\title{
Estado de desarrollo de las bibliotecas públicas cubanas: condiciones para la modelación matemática de su actividad
}

\author{
Emilio Setién Quesada*
}

\begin{abstract}
RESUMEN
Se presenta el estado actual de la base de datos estadísticos sobre la actividad de las bibliotecas públicas cubanas así como el nivel de desarrollo alcanzado por esas instituciones. Se analizan las posibilidades de aplicar a esos datos, modelos matemáticos que se apoyan en conceptos asequibles a la medición y la dirección en que debe orientarse esa aplicación. Se llega a conclusiones sobre la factibilidad de obtener un modelo matemático para representar el comportamiento peculiar de estas bibliotecas.
\end{abstract}

\section{ABSTRACT}

Based upon the statistical data bank, the current state of the activity of the public libraries is presented, as web as the level of development achieved by these institutions. The possibilities of applying mathematical models to this data is then analysed. These models are based upon available concepts of measurement and the direction that should guide this application. Conclusions are reached regarding the viability of obtaining a mathematical model to represent the peculiar behavior of these libraries.

$\boldsymbol{L}$ os estudios de la Bibliotecología sobre la actividad bibliotecaria se sustentan, entre otros, en el análisis de un profuso material fáctico, posible mediante una amplia utilización de la estadística, entendida ésta en su acepción de compilación de datos.

En análisis del estado actual de los datos estadísticos sobre la actividad de las bibliotecas públicas cubanas y la forma en que estos datos permiten reflejar el desarrollo de esa actividad constituye, por lo tanto, un paso fundamental para definir las posibilidades de aplicación de los modelos matemáticos que se apoyan en conceptos asequibles a la medición y para determinar la dirección en que debe orientarse esa aplicación.
Las bibliotecas públicas se definen en Cuba como instituciones que están al servicio de una comunidad, independientemente de su extensión y del tipo de usuario que atienden, y que poseen un fondo bibliotecario de carácter universal. ${ }^{1}$

Entre las condiciones a crear para la implantación del Sistema de Dirección de la Economía, aprobado por el I Congreso del Partido Comunista de Cuba, quedó señalada la necesidad de confeccionar y establecer un sistema de estadística económica adecuado para lograr que los centros de decisión puedan reaccionar con agilidad y fundamentación suficientes ante los cambios que se produzcan en el transcurso de la ejecución de los planes. ${ }^{2}$
En las condiciones del Socialismo. Como señala N. Krupskaia, los datos estadísticos son necesarios para que el Estado sepa si la población se sirve sistemáticamente de las bibliotecas y para saber con precisión hacia donde es necesario dirigir los esfuerzos con urgencia. ${ }^{3}$

Si se tiene en cuenta, además, que la aplicación de métodos matemáticos en las investigaciones bibliotecológicas tiene entre sus objetivos principales contribuir a determinar las proporciones de los elementos que integran la actividad bibliotecaria, se reafirma la necesidad de analizar la situación que presenta los datos estadísticos de las bibliotecas públicas en Cuba, así como las peculiaridades del desarrollo del sistema que ellos reflejan.

\footnotetext{
* Investigador Auxiliar de la Biblioteca Nacional de Cuba y Miembro de la Sociedad Cubana de Información Científica y Técnica y del Ejecutivo Nacional de la Asociación Cubana de Bibliotecarios
} 
En análisis que ahora se presenta, se circunscribe a la situación de los datos estadísticos de esas instituciones a partir del triunfo de la Revolución, puesto que los anteriores no se han podido recuperar en su totalidad hasta el presente; son objeto de búsqueda por las distintas bibliotecas del país en el marco de la línea de investigación denominada Origen y evolución de las bibliotecas públicas en Cuba. ${ }^{4}$

En 1960 las bibliotecas públicas que habían sido patrocinadas hasta el triunfo de la Revolución por la Organización $\mathrm{Na}$ cional de Bibliotecas Ambulantes y Populares (ONBAP), organización creada en 1954 bajo la tiranía de Batista, ${ }^{5}$ e intervenida por el Gobierno Revolucionario en 1959, fueron transferidas en número de 25 a la Dirección General de Cultura del Ministerio de Educación, según Ley
742 del 19 de febrero de $1960,{ }^{6}$ y se convirtieron, en 1961., en el núcleo inicial de la red de bibliotecas públicas atendidas por el entonces recién creado Consejo Nacional de Cultura.

Paulatinamente se fueron incorporando a esa red bibliotecas públicas sostenidas principalmente, antes de 1959, por los gobiernos provisionales y municipales, las cuales al igual que las procedentes de la ONBAP, fueron renovadas en su totalidad. Se adaptaron para ellas nuevos locales, se enriquecieron sus colecciones, y se inició la preparación del personal que las atendería a través de la actual Escuela Nacional de Técnicos de Bibliotecas del Ministerio de Cultura.

A la par, que se emprendió la creación de nuevas bibliotecas. Sin embargo, la cifra de bibliotecas públicas incorporadas a esa red hasta 1975 (un total de 120 bibliotecas)7, en comparación con la información que se ha podido reconstruir sobre las existentes en 1958 (123 bibliotecas)8, indica que el balance de los primeros quince años del periodo revolucionario, en la esfera de las bibliotecas públicas, puede ser considerado, hasta este momento, y desde el punto de vista cuantitativo, como un periodo de sustitución de las antiguas bibliotecas.

El crecimiento real de esas instalaciones se produjo, según los datos existentes, a partir de 1976, después de la constitución de los órganos del Poder Popular y coincidiendo con la creación del Ministerio de Cultura, durante el proceso de institucionalización del país que se inició después del I Congreso del Partido Comunista de Cuba.

Tabla 1. Crecimiento de las bibliotecas públicas en Cuba después de 1958

\begin{tabular}{|l|l|l|l|l|l|l|l|l|l|}
\hline 1958 & $123^{9}$ & 1963 & $26^{10}$ & 1968 & 50 & 1973 & 94 & 1978 & 142 \\
\hline 1959 & 123 & 1964 & 31 & 1969 & 51 & 1974 & 107 & 1979 & 165 \\
\hline 1960 & 123 & 1965 & 35 & 1970 & 50 & 1975 & 120 & 1980 & 195 \\
\hline 1961 & 123 & 1966 & 41 & 1971 & 52 & 1976 & 128 & 1981 & 222 \\
\hline 1962 & 123 & 1967 & 43 & 1972 & 76 & 1977 & 134 & 1982 & 243 \\
\hline
\end{tabular}

En el título Series cronológicas sobre cultura y arte se ofrece, además, información sobre otros indicadores del comportamiento de las bibliotecas públicas.

Tabla 2. Indicadores del comportamiento de las bibliotecas públicas cubanas ${ }^{11}$

\begin{tabular}{|c|c|c|c|c|}
\hline Año & $\begin{array}{c}\text { Fondo bibliotecario } \\
\text { miles }\end{array}$ & $\begin{array}{c}\text { Préstamos } \\
\text { miles }\end{array}$ & $\begin{array}{c}\text { Lectores } \\
\text { miles }\end{array}$ & $\begin{array}{c}\text { Bibliotecarios } \\
\text { unidades }\end{array}$ \\
\hline 1963 & & & 399.0 & \\
\hline 1965 & 841,1 & & 1126,1 & \\
\hline 1966 & 601,2 & & 1718,0 & \\
\hline 1967 & & & 1623,4 & \\
\hline 1970 & 1978,4 & 1597,6 & 2161,2 & \\
\hline 1971 & & 1792,8 & 2505,7 & \\
\hline 1972 & 1383,3 & 2037,1 & 2550,1 & \\
\hline 1973 & 1561,3 & 2306,0 & 2809,8 & \\
\hline 1974 & 1598,0 & 2783,0 & 2995,6 & \\
\hline 1979 & 2638,8 & 3998,0 & 5326,9 & 1459 \\
\hline 1980 & 2718,9 & 4540,6 & 5268,7 & 1449 \\
\hline 1981 & 3076,3 & 6925,6 & 7222,0 & 1633 \\
\hline 1982 & 4085,1 & 8093,7 & 8724,4 & \\
\hline
\end{tabular}


Como se puede apreciar, la cantidad de préstamos es mayor que la cantidad de fondo bibliotecario en la casi totalidad de los años reportados, lo que corresponde, según el análisis efectuado por Ju. N. Stoljarov, con el comportamiento de las bibliotecas públicas. ${ }^{12}$

Los indicadores reflejados en la tabla 2 representan los elementos esenciales de la actividad bibliotecaria: fondo bibliotecario, préstamo, lectores y bibliotecarios.

En el conjunto de modelos matemáticos localizados a través de la bibliografía consultada, los indicadores de uso más frecuente son el fondo bibliotecario y los préstamos. La mayoría de los modelos que utilizan estos indicadores lo hacen con el fin de elaborar pronósticos sobre el crecimiento de los fondos y sobre el comportamiento de su utilización, para lo que emplean datos históricos que reflejan tendencias relativamente estables, susceptibles de ser modeladas mediante distintos métodos matemáticos.

A continuación se analizan, a manera de ejemplo, algunos de estos modelos, que fueron elegidos teniendo en cuenta que sus creadores se encuentran entre los autores más citados en la literatura sobre aplicación de modelos matemáticos a la actividad bibliotecaria.

En el artículo titulado "Analytical Models for Library Planning”, Ferdinand Leimkuhler presenta un modelo matemático simple para pronósticos del crecimiento del fondo. Este modelo se basa en la evidencia empírica del comportamiento de bibliotecas, estudiadas por ese autor, en las que el conocimiento del fondo bibliotecario se produce a razón de tasas relativamente estables. $^{13}$

Sin embargo, en el caso de las bibliotecas públicas cubanas, el crecimiento anual del fondo bibliotecario no se ajusta significativamente a las tasas medias que se pueden estimar de acuerdo con datos históricos, lo que puede comprobarse mediante el análisis de los datos que ofrece la tabla 2 , que corresponden al sistema de bibliotecas públicas cubanas en su conjunto, y por el comportamiento particular de las distintas bibliotecas que lo integran, como se aprecia en los ejemplos siguientes:

Tabla 3 Ejemplos de crecimiento del fondo en bibliotecas del sistema de 1981 a $1984 .^{14}$

\begin{tabular}{|c|c|c|c|c|}
\hline \multirow{4}{*}{} & \multicolumn{3}{|c|}{ Bibliotecas } \\
\cline { 2 - 5 } & $\boldsymbol{A}$ & $\boldsymbol{B}$ & $\boldsymbol{C}$ & $\boldsymbol{C H}$ \\
\hline Crecimiento anual esperado, a las tasas constantes 1981-1984 & 459 & 713 & 1199 & 742 \\
\hline Crecimiento anual real & \multicolumn{4}{|c|}{} \\
\hline $1981-1982$ & 801 & 1106 & 1258 & 1066 \\
\hline $1982-1983$ & 10 & 210 & 666 & 649 \\
\hline $1983-1984$ & 565 & 824 & 1674 & 511 \\
\hline
\end{tabular}

Estos datos, evaluados mediante la prueba de X2, muestran que la tasa media anual de crecimiento del fondo del trienio no presenta, en general, buen ajuste con respecto a las tasas anuales del crecimiento real, por lo que puede afirmarse que ese crecimiento no se realiza a tasas estables. ${ }^{15}$

Algo similar a lo señalado sobre comportamiento de los fondos ocurre con respecto al uso de los documentos. Leimkahler y otros autores citados por él, como son Fussler, Simon y Jain, sostienen la posibilidad de identificar tasas constantes de desuso del fondo por obsolescencia. Sobre esta base elaborar modelos matemáticos para estimar los niveles que pueden alcanzar los préstamos en las biblioteca. ${ }^{16}$ No obstante, la realidad de las bibliotecas públicas cubanas no permite corroborar ese supuesto.
Dieciséis muestras empleadas en un estudio sobre la utilización del fondo de esas instituciones, en las que se incluyeron 715 documentos agrupados en distintas temáticas y que reflejan los préstamos de los últimos 15-20 años, ofrecieron, en todo caso, resultados tales como:

- Tasa media anual estimada de decrecimiento del uso de documentos: $17 \%$

- Tasas reales de decrecimiento anual: $11 \%, 17 \%, 25 \%, 50 \%, 60 \%, 67 \%{ }^{17}$

Por otra parte, en el artículo "A Simple Stochastic Model for Library Loans, ${ }^{18}$ de Quentin L. Burrell, este autor propone un modelo basado en la distribución geométrica simple, esto es, en aquella distribución en que, como define Firthorne, la variable independiente crece geométricamente, mientras que la dependiente crece aritmétricamente. ${ }^{19}$ Este modelo es el siguiente:

\section{$f_{r}(T)=f p q^{r}$ para $r=0,1,2$}

Donde:

$\mathrm{f}_{\mathrm{r}}(\mathrm{T})=$ cantidad de volúmenes prestados $r$ veces durante un periodo de tiempo determinado $\mathrm{T}$;

$\mathrm{f}=$ cantidad total de volúmenes disponibles para el préstamo durante ese periodo;

$\mathrm{p}=$ probabilidad de que los volúmenes utilizados se presten una sola vez cada uno;

$\mathrm{q}^{\mathrm{r}}=$ probabilidad de que los volúmenes utilizados se presten $r$ veces cada uno.

Para la aplicación práctica del modelo, Burrell trabaja con las fórmulas:

I) $\mathrm{E} 1=\left(\mathrm{f}-\mathrm{f}_{\mathrm{o}}\right) \mathrm{p}$

II) $\mathrm{E}_{\mathrm{r}}-1=\mathrm{Q} \mathrm{E}_{\mathrm{r}}$, para $\mathrm{r}=1,2, \ldots$

En la fórmula I 
$\mathrm{E}_{\mathrm{r}}=$ cantidad de volúmenes que se prestan una vez cada uno durante el periodo estudiado;

$\mathrm{f}=$ cantidad total de volúmenes disponibles para el préstamo en ese periodo;

$\mathrm{f}_{\mathrm{o}}=$ cantidad total de volúmenes no utilizados durante dicho periodo; $\mathrm{p}=\left(\mathrm{f}-\mathrm{f}_{\mathrm{o}}\right) / \mathrm{m}$, siendo $\mathrm{m}$ el total de préstamos en el periodo estudiado.

En la fórmula II

$\mathrm{E}_{\mathrm{r}}+1=$ estimado de la cantidad de volúmenes prestados $r$ veces para $r=1,2, \ldots$

$\mathrm{q}=1-\mathrm{p}$
La aplicación de este método a cinco muestras tomadas de la realidad de las bibliotecas públicas cubanas ofreció los resultados que se reflejan en la tabla siguiente:

Tabla 4.- Estimado de volúmenes según frecuencia de préstamos, mediante la aplicación del método propuesto por Burrell a bibliotı cas públicas cubanas (datos de 1985).

\begin{tabular}{|c|c|c|c|}
\hline Muestras & $\begin{array}{c}\text { Frecuencia de préstamos } \\
\text { por volumen }\end{array}$ & $\begin{array}{c}\text { Estimado de cantidad de } \\
\text { volúmenes en cada } \\
\text { frecuencia }\end{array}$ & $\begin{array}{l}\text { Cantidad real de } \\
\text { volúmenes en } \\
\text { cada frecuencia }\end{array}$ \\
\hline \multirow[t]{7}{*}{ Muestra 1} & 1 & 62 & 75 \\
\hline & 2 & 30 & 22 \\
\hline & 3 & 14 & 8 \\
\hline & 4 & 7 & 3 \\
\hline & 5 & 3 & 3 \\
\hline & 6 & 2 & 4 \\
\hline & 70 más & 1 & 4 \\
\hline \multirow[t]{7}{*}{ Muestra 2} & 1 & 56 & 75 \\
\hline & 2 & 26 & 13 \\
\hline & 3 & 12 & 4 \\
\hline & 4 & 5 & 3 \\
\hline & 5 & 3 & 0 \\
\hline & 6 & 1 & 2 \\
\hline & 7 o más & 1 & 7 \\
\hline \multirow[t]{6}{*}{ Muestra 3} & 1 & 18 & 22 \\
\hline & 2 & 9 & 8 \\
\hline & 3 & 5 & 4 \\
\hline & 4 & 3 & 0 \\
\hline & 5 & $I$ & 1 \\
\hline & 60 más & 1 & 3 \\
\hline \multirow[t]{7}{*}{ Muestra 4} & 1 & 24 & 36 \\
\hline & 2 & 13 & 5 \\
\hline & 3 & 7 & 2 \\
\hline & 4 & 4 & 1 \\
\hline & $\cdots$ & 2 & 1 \\
\hline & 6 & 1 & 3 \\
\hline & 7 o más & 1 & 5 \\
\hline \multirow[t]{7}{*}{ Muestra 5} & 1 & 35 & 46 \\
\hline & 2 & 18 & 11 \\
\hline & 3 & 9 & 5 \\
\hline & 4 & 5 & 3 \\
\hline & 5 & 3 & 1 \\
\hline & 6 & 1 & 2 \\
\hline & 70 más & 1 & 5 \\
\hline
\end{tabular}


Estos datos, evaluados mediante la prueba de $\mathrm{X}^{2}$, indican que la distribución de volúmenes según la frecuencia de préstamos, estimada de acuerdo con el modelo de Burell, no presenta en general buen ajuste con la base de datos reales tomada de las bibliotecas públicas cubanas, entre otros, porque los volúmenes muy productivos (de mucho préstamo) no presentan ese ajuste con respecto a la distribución lograda con el modelo.

Los resultados alcanzados por Burell al aplicar su modelo a la realidad de países desarrollados (Gran Bretaña, específicamente) pueden apreciarse en el ejemplo siguiente:

Tabla 5 Estimado de volúmenes según frecuencia de préstamos, presentado por Burell. ${ }^{20}$ Muestra

\begin{tabular}{|c|c|c|}
\hline $\begin{array}{c}\text { Frecuencia de } \\
\text { préstamos por } \\
\text { volumen }\end{array}$ & $\begin{array}{c}\text { Estimado de } \\
\text { cantidad de vo- } \\
\text { lúmenes en } \\
\text { cada frecuencia }\end{array}$ & $\begin{array}{c}\text { Cantidad real } \\
\text { de volúmenes } \\
\text { en cada fre- } \\
\text { cuencia }\end{array}$ \\
\hline 1 & 61 & 65 \\
\hline 2 & 30 & 26 \\
\hline 3 & 15 & 12 \\
\hline 4 & 8 & 10 \\
\hline 5 & 4 & 5 \\
\hline 6 & 2 & 3 \\
\hline 7 o más & 1 & 1 \\
\hline
\end{tabular}

En este caso la aplicación de $\mathrm{X}^{2}$ permite aceptar un buen ajuste entre los valores estimados y los reales para $\mathrm{X}^{2} 0.05$ con 6 grados de libertad.

En conclusión, los resultados de la aplicación del modelo de Burell a la realidad cubana no se ajustan a las tendencias que se presentan en la distribución según frecuencia de préstamos por volumen.

Antes de abandonar el análisis del modelo de Burell, debe subrayarse que su aplicación reclama, para cada periodo estudiado, el conocimiento de:

a) cantidad de volúmenes disponibles para el préstamo en el periodo;

b) la cantidad de préstamos efectuados en ese periodo;

c) cantidad de volúmenes realmente utilizados durante dicho periodo.
Las informaciones descritas en a y b se compilan sistemáticamente por las bibliotecas en general, y por las bibliotecas públicas cubanas, en particular. Sin embargo, en las condiciones organizativas actuales de estas últimas, la información descrita en c no se compila sistemáticamente.

Por otra parte, el análisis comparado de esas bibliotecas, tomando como base la cantidad de documentos en el fondo y la cantidad de bibliotecarios por lector potencial, así como la composición cualitativa de esa fuerza de trabajo y su productividad, presenta la situación que muestran los ejemplos siguientes:

Tabla 6 Índices de volúmenes y bibliotecarios por lector potencial. ${ }^{21}$

\begin{tabular}{|c|c|c|}
\hline $\begin{array}{c}\text { Biblioteca } \\
\text { (ejemplos) }\end{array}$ & $\begin{array}{c}\text { Volúmenes por } \\
\text { lector potencial }\end{array}$ & $\begin{array}{c}\text { Bibliotecarios } \\
\text { por lectores po- } \\
\text { tenciales }\end{array}$ \\
\hline $\mathrm{A}$ & 0,27 & $1 \times 3177$ \\
\hline $\mathrm{B}$ & 0,81 & $1 \times 6255$ \\
\hline $\mathrm{C}$ & 0,58 & $1 \times 5412$ \\
\hline $\mathrm{Ch}$ & 0,32 & $1 \times 5394$ \\
\hline $\mathrm{D}$ & 0,77 & $1 \times 2524$ \\
\hline $\mathrm{E}$ & 0,44 & $1 \times 3957$ \\
\hline $\mathrm{F}$ & 0,37 & $1 \times 8685$ \\
\hline
\end{tabular}

Tabla 7 Categorías del personal técnico en las plantillas de las bibliotecas y su rendimiento. ${ }^{22}$

\begin{tabular}{|c|c|c|c|c|}
\hline $\begin{array}{c}\text { Biblio- } \\
\text { teca }\end{array}$ & $\begin{array}{c}\text { Espe- } \\
\text { cialistas }\end{array}$ & $\begin{array}{c}\text { Técni- } \\
\text { cos } \\
\text { medios }\end{array}$ & $\begin{array}{c}\text { Auxiliares } \\
\text { de Infor- } \\
\text { mación }\end{array}$ & $\begin{array}{c}\text { Préstamos } \\
\text { por biblio- } \\
\text { tecario }\end{array}$ \\
\hline A & & 3 & & 6373 \\
\hline B & 1 & 1 & & 15336 \\
\hline C & & 2 & 2 & 11535 \\
\hline Ch & & 2 & 2 & 6124 \\
\hline D & 1 & 2 & 3 & 5122 \\
\hline E & 1 & 3 & & 4758 \\
\hline F & & 2 & & 10868 \\
\hline
\end{tabular}

Como puede apreciarse por las tablas que anteceden todas las bibliotecas no tienen la misma proporción de documentos, ni de bibliotecarios con respecto a la cantidad de lectores a atender. Aquellas que cuentan con proporciones similares en la relación bibliotecarios-lectores (ejemplos A y E), no siempre presentan la misma composición de categorías de personal bibliotecario en sus plantillas.
Algunas están atendidas por especialistas, técnicos medios y auxiliares de información, otras, por técnicos medios y auxiliares; y otras, sólo por técnicos medios, o por auxiliares de información (aunque este último caso no se manifiesta en los ejemplos presentados).

Los factores combinados, mencionados anteriormente, implican distintos costos por concepto de salario. En Cuba, los salarios representan entre el 70 y el $80 \%$ de los gastos de las bibliotecas, por lo que constituyen el componente principal de los costos de operación de esas instituciones. ${ }^{23}$ El rendimiento por bibliotecario varía marcadamente de una biblioteca a otra, lo que, unido a las diferencias salariales que se originan debido a las distintas formas de composición de las plantillas, conduce a variaciones en los costos por préstamo efectuado.

El propio avance de las entidades que integran el sistema de bibliotecas públicas del país conduce a que varíe la composición cualitativa de sus plantillas de un periodo a otro, lo que se refleja en la incorporación de personal técnico más calificado, como expresión de desarrollo.

De los ejemplos anteriores se infiere que el comportamiento de los elementos esenciales de la actividad bibliotecaria, susceptibles de medición, según se reflejan en los datos estadísticos de las bibliotecas públicas cubanas, corresponden a un proceso todavía no estabilizado, lo que limita la posibilidad de aplicar modelos matemáticos al pronóstico de su comportamiento.

Consecuentemente la modelación matemática de la actividad de esas bibliotecas ha de orientarse hacia el análisis de modelos matemáticos que permitan elaborar generalizaciones sobre lo ya ocurrido en esas instituciones y que permitan también comparar a unas bibliotecas con otras, en distintos periodos, con el fin de orientar los esfuerzos hacia la reducción de las diferencias significativas que existen entre ellas hasta límites aceptables.

Se observa, también, que en el comportamiento de las bibliotecas públicas cubanas pesa más la singularidad que la generalidad, lo que obliga a pensar en modelos que permitan reflejar esa singularidad mediante el empleo del mayor número de elementos posibles, siempre 
en el marco de aquellos que sean esenciales para la actividad bibliotecaria.

El análisis comparado entre las distintas bibliotecas que integran el sistema, con el fin de orientar su desarrollo, resulta complejo, debido a los diferentes elementos y relaciones que deben ser considerados y comparados simultáneamente. Pudiera asumirse que algunos de esos elementos y relaciones son más significativos que otros, con lo que el problema quedaría reducido a la comparación de los valores de esos elementos y relaciones, pero esto implicaría el reconocimiento de que otros elementos y relaciones de la actividad bibliotecaria, concebida como un sistema, quedarían reducidos a la condición de no significativos, lo que no puede aceptarse, puesto que todos lo son, en tanto definen integralmente la esencia que diferencia a este sistema de otros.

Circunstancialmente, algunos elementos y relaciones pudieran considerarse más importantes que otros, para el sistema, por el rol que desempeñan en su equilibrio interno, o en su equilibrio con el medio, pero esta circunstancia no reduce a los restantes a la condición de no significativos. Entonces, es necesario que en el modelo que se emplee para el análisis y comparación de las bibliotecas públicas del país estén presentes todas las características esenciales de la actividad bibliotecaria.

Estas consideraciones se sustentan en los criterios que expone al respecto Douglas $\mathrm{H}$, Rothenberg sobre la integralidad de los sistemas informativos y su moderación, en el artículo “An Efficiency Model and Performance Function for Information Retrieval Systems". ${ }^{24}$

Entre las medidas estadísticas que posibilitan la comparación de unas cosas con otras y el conocimiento de cuánto esas cosas cambian se encuentran los números índice los que, como señala Freund, se utilizan ampliamente en las ciencias sociales. ${ }^{25}$

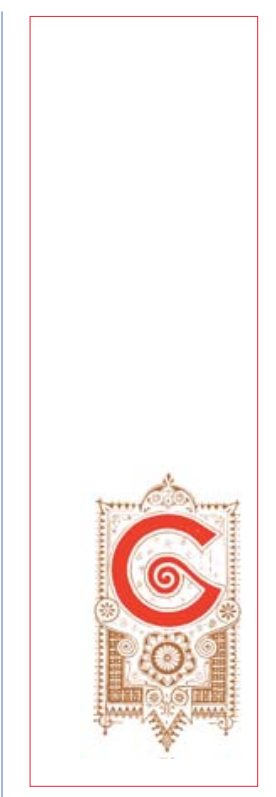

Spiegel define a los números índice como la medida estadística que tiene por objeto mostrar los cambios en una variable o en un grupo de variables, relacionadas entre sí, con respecto al tiempo, a lugares y a otras características. ${ }^{26}$

Entonces, como el tratamiento matemático de los datos estadísticos que controlan sitemáticamente las bibliotecas públicas cubanas permite elaborar distintos índices que en su conjunto expresan la eficiencia de esa actividad, y como los números índice permiten analizar de manera integral el comportamiento de una cosa o de un conjunto de cosas en el transcurso del tiempo, y mostrar los cambios que se producen en un grupo de variables relacionadas entre sí con respecto al tiempo y también en situaciones y lugares diferentes, se concluye que este tipo de medida estadística es aconsejable para su aplicación a la realidad actual de las bibliotecas públicas cubanas, lo que satisface la séptima hipótesis de esta investigación y constituye la base sobre la que se orienta este trabajo de aquí en adelante.

Por último, debe considerarse que la actividad bibliotecaria comprende también el conjunto de disposiciones estatales y sociales que propician el uso del fondo bibliotecario como propiedad colectiva. En las condiciones del socialismo esas disposiciones están comprendidas en las orientaciones partidistas para el desarrollo de la actividad bibliotecaria, en los planes estatales que guían ese desarrollo, en la normación y la legislación vigentes sobre la actividad bibliotecaria.

En los documentos mencionados se expresan los niveles que la sociedad se propone alcanzar en el desarrollo de dicha actividad. Varios de los documentos contenidos en documentos cubanos de este tipo pueden ser expresados en conceptos asequibles a la medición, y guardan relación con los elementos y relaciones esenciales de la actividad bibliotecaria descritos en este trabajo. Entre ellos se encuentran: los volúmenes y el personal bibliotecario que deben tener las bibliotecas con respecto a la población a servir y los niveles de rendimiento de ese personal. ${ }^{27}$ 
Entonces, de acuerdo con los razonamientos antes expuestos, puede afirmarse que existe la posibilidad de:

Obtener un modelo matemático que permita reflejar los elementos y relaciones esenciales de la actividad bibliotecaria, según se manifiestan en las bibliotecas públicas cubanas, que ofrezca, al mismo tiempo, un índole integral de comportamiento de estas instituciones, para ordenarlas y compararlas entre sí, en relación con las disposiciones estatales vigentes para la orientación de ese desarrollo.

La comprobación de esta posibilidad será objeto de un próximo trabajo.

\section{NOTAS BIBLIOGRÁFICAS}

1. NC 39-03-82, Sistema de Información y Documentación Bibliográfica. Actividad científico informativa. Términos y definiciones. Op. cit. en bibliografía.

2. PARTIDO COMUNISTA DE CUBA. Congreso, $1^{\circ}$., La Habana, 1975. Tesis y resoluciones del Primer Congreso del Partido Comunista de Cuba. Op. cit. en bibliografía, p. 205.

3. KRUPSKAIA, N.K. O bibliotechnom dele. Op. cit. en bibliografía, p. 274.

4. Encuentro Científico Biblotecológico, $3^{\circ}$., La Habana, 1983. Reseña. Op. cit. en bibliografía.

5. Cuba, Leyes, Decretos, etc. Ley Decreto no. 1810, op. cit. en bibliografía pp. 22233-36.

6. Cuba, Leyes, Decretos, etc. Ley No. 742, Op. cit. en bibliografía, pp. 4531-32.

7. CUBA, Comité Estatal de Estadísticas. Series cronológicas de cultura y arte. Op. cit., en bibliografía, pp. 25-27.

8. SETIÉN, Emilio. El desarrollo de la Biblioteca Nacional durante 75 años y su influencia en el movimiento bibliotecario del país, Op. cit., en bibliografía, pp. 59-95.

9. Ibid

10. CUBA, Comité Estatal de Estadísticas. Series cronológicas de cultura y arte, Op. cit., en bibliografía, p. 27.

11. C UBA, Comité Estatal de Estadísticas. Series cronológicas de cultura y arte. Op. cit., en bibliografía, pp. 25-27.

12. STOLJAROV, Ju. N. Amplitud óptima de los fondos de bibliotecas públicas, op. cit. en bibliografía, pp. 23-29.

13. LEIMKULER, F. Analytical Models for Library Planning, op. cit. en bibliografía, pp. 390-398.

14. CUBA, Ministerio de Cultura. Dirección de Bibliotecas. Datos para el pronóstico al 2005, op. cit. en bibliografía.

15. SPIEGEL, Murria r. Teoría y problemas de estadística, op. cit. en bibliografía, pp. 190-191, 345 .

16. KEIMHULER, Ferdinand F. Analytical Models for Library Planning, op. cit. en bibliografía, pp. 390-398.

17. DÍAZ Páez Carballo. A. Y E. Yera Barrera. Obsolescencia del fondo de la Biblioteca Pública Rubén Martínez Villena, op. cit. en bibliografía.

18. BURRELL, Quentin. A simple Stochastic Model for Library Loans, op. cit. en bibliografía, pp. 115-132. 
19. FIRTHORNE, Robert. Empirical Hyperbolic Distributions for Bibliometric description and Prediction, op. cit., en bibliografía, pp. 521-534.

20. BURELL, Quentin. A simple Stochastic Model for Library Loans, op. cit., en bibliografía, pp. 115-132.

21. CUBA. Ministerio de Cultura. Dirección de Bibliotecas. Datos para el pronóstico al 2005. op. cit. en bibliografía.

22. C UBA. Ministerio de Cultura. Dirección de Bibliotecas. Datos para el pronóstico al 2005. op. cit. en bibliografía.

23. C UBA. Ministerio de Cultura. Dirección de Bibliotecas. Circular sobre el presupuesto de las bibliotecas públicas., op. cit., en bibliografía.

24. ROTHENBERG, Douglas H. An efficiency Model and a performance function for an Information Retrieval System., op. cit., en bibliografía, pp. 707-714.

25. FREUND, John E. Estadística elemental moderna, op. cit., en bibliografía, p. 118.

26. SPIEGEL, Murria R. Teoría y problemas estadísticos..., op. cit., en bibliografía, p. 313.

27. CUBA. Ministerio de Cultura. Dirección de Bibliotecas. Pronóstico hasta el año 2000. op. cit. en bibliografía. NRCU 007:80 Bibliotecas Públicas. Servicios Índices y métodos para el control de la calidad y el rendimiento., op. cit., en bibliografía.

\section{BIBLIOGRAFÍA}

BURELL, Quentin L., A Simple Stochastic Model for Library Loans. Journal of Documentation (London) 36(2): 115-132: June, 1980.

CUBA. Comité Estatal de Estadísticas Series cronológicas de cultura y arte. - [Ciudad de La Habana : s.n.]1986. -60 p.

CUBA. Leyes, decretos, etc., Ley decreto 1810. Gaceta Oficia (Habana) 52(275):22233-36; 26 noviembre 1954.

—. Ley No. 742. Gaceta Oficial (Habana) 54(4):4531-32; 23 febrero 1960.

CUBA. Ministerio de Cultura. Dirección de Bibliotecas. Circular sobre el presupuesto de las bibliotecas públicas. - Ciudad de La Habana, 1984. - 2 h.

-.Datos para el pronóstico al 2005. — [1985] no publicado.

—. Pronóstico hasta el 2000. .- [1979] - 12 h. - no publicado

DÍAZ Páez Carballo, Aix y Erik Yera Barrera. Obsolescencia del fondo de la Biblioteca Pública. Rubén Martínez Villena. - 1985. - 29 h. Mecanografiado Tutor: Lic. Emilio Setién En la portada: Ciudad de La Habana Proyecto de grado, Escuela Nacional de Técnicos de Biblioteca.

Encuentro Científico Bibliotecológico, $3^{\circ}$., La Habana, 1983. Reseña. - Ciudad de La Habana : Dirección de Bibliotecas del Ministerio de Cultura, 1983. - 42 h.

FIRTHORNE, Robert. "Empirical Hyperbolic Distributiones (Bradford Zipf-Mandelbrot) for Bibliometric Description and Prediction". - En Saracevic, Tefko, comp. Introduction to Information Sciences. - New York : Bowker, 1970. - pp. 521-534. 
FREUND, John E. Estadística elemental moderna. [La Habana : Instituto del Libro, 1972]- 466 p.

KRUPSKAIA, NK. O bibliotechnom dele. -Moskova : [s.n.], 1957. - 714 s.

LEIMKIHLER, Ferdinand y Michael D. Cooper. Analytical Models for Library Planning. Journal of the American Society for Information Sciences (Washington) 22(6):390-398; December, 1971.

NC 39-03-82. Sistema de Información y Documentación Bibliográfica. Actividad científico informativa. Términos y definiciones. Vig. Desde 83,4. -8 p.

NRCU 008-80. Bibliotecas públicas. Servicios: Índices y métodos para el control de calidad y el rendimiento. - Vig. Desde 81.9.-1 p.

PARTIDO COMUNISTA DE CUBA, congreso, $1^{\circ}$., La Habana, 1975. Tesis y resoluciones del Primer Congreso del Partido Comunista de Cuba. - La Habana : Dep. de Orientación Revolucionaria del Comité Central del Partido Comunista de Cubam 1976. - 675 p.

ROTHENBERG, Douglas M. "An Efficiency Model and a Performance Function for an Information Retrieval System". - En Saracevic, Tefki, comp. Introduction to Information Sciences. - New York : Bowker, 1970. - pp. 707-714.

SETIEN Quesada, Emilio. "El desarrollo de la biblioteca nacional durante 75 años y su influencia en el movimiento bibliotecario del país”. En Revista de la Biblioteca Nacional José Martí (La Habana) 68(1): 59-95; enero-abril, 1977.

SPIEGEL, Murria R. Teoría y problemas de estadística : 875 problemas resueltos. - La Habana : Editorial Pueblo y Educació, 1977. $-358 \mathrm{p}$.

STOLJAROV, Ju. N. "Amplitud óptima de los fondos de las bibliotecas públicas”. En Boletín de la UNESCO para las bibliotecas (París) 27(1): 23-29; enero-febrero, 1973. 